\title{
Drying Kinetics of a Porous Spherical Particle and the Inversion Temperature
}

\author{
S. Messai, ${ }^{1}$ J. Sghaier, ${ }^{2}$ D. Lecomte, ${ }^{3}$ and A. Belghith ${ }^{1}$ \\ ${ }^{1}$ Laboratoire d'Energétique et des Transferts Thermiques et Massiques, Faculté des Sciences de \\ Tunis, Campus universitaire, Tunis, Tunisie \\ ${ }^{2}$ Département d'Energétique, Ecole Nationale d'Ingénieurs de Monastir, Monastir, Tunisie \\ ${ }^{3}$ Centre RAPSODEE, Ecole des mines d'Albi-Carmaux, Campus Jarlard, Albi CT, France
}

\begin{abstract}
A model is presented for drying of a single porous particle with superheated steam and humid air. Experimental data for spherical porous ceramic particle reported in the literature were used for the validation of the model. An inversion temperature at which the evaporation rates within superheated steam and humid air are equal was predicted. The effect of thermophysical properties of the particle (permeability $10^{-14}-10^{-17} \mathrm{~m}^{2}$, diameter $3 \times 10^{-3}-10 \times 10^{-3} \mathrm{~m}$ ) and operating variables (gas mass flux $0.26-0.78 \mathrm{~kg} \mathrm{~m}^{-2} \mathrm{~s}^{-1}$, drying agent temperature $120-200^{\circ} \mathrm{C}$ ) is tested. The inversion temperature is shown to be affected by the thermophysical properties of the porous particle and of the drying agent.
\end{abstract}

Keywords Drying; Humid air; Inversion temperature; Porous particle; Superheated steam

\section{INTRODUCTION}

Superheated steam drying is an attractive technology that offers potential advantages such as reduced energy cost, smaller equipment, reduced emissions, reduced fire and explosion hazards, and improvements in product quality. ${ }^{[1-4]}$ It can replace the air drying except for the thermally sensitive products.

Wenzel and White ${ }^{[5]}$ and Chu et al. ${ }^{[6]}$ were the first to show experimentally that more intensive evaporation occurs in superheated steam than in air. Yoshida and Hyodo $^{[7]}$ demonstrated that superheated steam can provide an excellent medium for drying food products. Compared to humid air, superheated steam is cleaner, provides higher evaporation rate and there is less oxidation in food, thus reducing the loss in nutritional value during the drying process. The authors showed experimentally that the curves of evaporation rate with air and superheated steam would intersect at a point called the inversion temperature.

Correspondence: J. Sghaier, Département d'Energétique, Ecole Nationale d'Ingénieurs de Monastir, Avenue Ibn Eljazzar, 5019 Monastir, Tunisie; E-mail: Jalila.Sghaier@enim.rnu.tn
Above this temperature, water evaporation rate increases as the humidity of the air increases with a maximum for pure superheated steam. The change in drying rate with the gas flux and the estimation of the inversion temperature has been the subject of various theoretical and experimental investigations.

Trommelen and Crosby ${ }^{[8]}$ investigated numerically and experimentally the evaporation of water into dry air, humid air, and superheated steam. They studied the evaporation rate and the inversion temperature in a free stream. Schwartze and Bröcker ${ }^{[9]}$ presented a comparative table summarizing previous theoretical and experimental results giving the inversion temperature between 160 and $260^{\circ} \mathrm{C}$, depending on the fluid flow regime (turbulent or laminar). They carried out a theoretical study of water evaporation and introduced refined definitions of the inversion temperature. The apparent inversion temperature was defined as the temperature at the beginning of the evaporation area for which the average evaporation rates into two gas fluxes with different vapor mole fractions are equal. These new definitions enabled a more precise description of the inversion temperature phenomenon and explained the reasons for discrepancies in inversion temperature values obtained in earlier studies. Costa and Neto da Silva ${ }^{[10]}$ studied the evaporation of water from a wet film into a gaseous stream of dry air, moist air, and superheated steam. They demonstrated that the numerical values of the local and effective inversion temperature were strongly dependent on the local heat and mass transfer and thus on the correlations used to calculate it. They proposed analytical expressions for the inversion temperature.

The usual models to describe the inversion temperature consider a wet planar surface that releases free water into a stream of humid air or superheated steam. In spite of the simplicity of these assumptions, there are differences between the inversion temperature values reported in the literature ${ }^{[9,10]}$ For a porous particle, the determination of 
the inversion temperature is even more complex than for a planar wet surface because of the porous structure and geometry. In the literature, few papers investigated the inversion temperature based on the heat and mass transfer during the drying of a porous particle. In their numerical study, Chen et al. ${ }^{[1]}$ assumed that the removal of water proceeds with the formation of a wet-dry interface and a receding front with time. For coal particles, they studied the effects of gas mass flux, particle radius and permeability on the inversion temperature. The coal inversion temperature was shown to have a minimum as gas mass flux and particle size increase. With the increase of coal permeability, the inversion temperature decreases. Suvarnakuta et al. ${ }^{[12]}$ presented a comparative study of low-pressure superheated steam and vacuum drying of molecular sieve beads porous particle. They investigated the effect of the operating pressure on the inversion temperature. They also showed the difference between the inversion temperature calculated from the constant rate period and the falling rate period. Shibata ${ }^{[13]}$ investigated the drying process of porous sintered glass beads, baked clay, and cemented glass balloons in both steam and air under low pressure. The author showed the influence of pressure on the normalized drying rate and critical moisture content for the two cases. However, the inversion temperature of these products was not mentioned in his study.

In spite of the abundant literature on ceramic drying, there is little information on the inversion temperature of porous ceramic particle. Tatemoto et al. ${ }^{[14]}$ reported an inversion temperature value of $160^{\circ} \mathrm{C}$ for a porous ceramic plate dried with superheated steam and hot air, without mentioning the effect of the external (drying fluid velocity) and internal (particle diameter and permeability) parameters on the inversion temperature.

The calculation of the inversion temperature is of major importance for the design of a drying process. Although superheated steam is chosen as drying agent for its safety and energy efficiency properties, larger heat transfer rates with respect to conventional air drying can only be achieved for temperatures higher than the inversion temperature.

The objective of the present study is to predict numerically the inversion temperature using a model of heat and mass transfer in a porous particle during superheated steam and humid air drying. Unlike previous models, we have used the volume averaging ${ }^{[15-17]}$ and a dichotomy method to calculate the evaporation rate. The model is then validated with the experimental data of a single porous ceramic drying. ${ }^{[18,19]}$ Hence, the influence of external and internal parameters on the inversion temperature is also investigated.

A comparison between our model and that of Chen et al. ${ }^{[11]}$ for coal inversion temperature is presented.

\section{FORMULATION}

The spherical porous particle is made of an inert and rigid solid matrix containing a liquid phase and a gaseous phase. Hence, the particle is a discontinuous medium. The theoretical formulation of heat and mass transfer in the porous particle is obtained by a change of scale. ${ }^{[15-17]}$ At microscopic scale, the size of the representative volume $\mathrm{V}$ is small with respect to the pore sizes. At macroscopic scale the size of the representative volume is large with respect to the pore sizes. The change of scale allows converting the real discontinuous medium to an equivalent fictional continuous one.

\section{Governing Equation of Heat and Mass Transfer}

The macroscopic differential equations are obtained by taking the average of the microscopic equations over the averaging volume and using closing assumptions. Several simplifying assumptions are made in order to obtain a closed set of macroscopic governing equations:

- the particle is from the macroscopic point of view homogeneous and isotropic

- local thermal equilibrium is assumed

- compression work and viscous dissipation are neglected

- the dispersion and tortuosity terms are modeled as diffusion fluxes.

The initial temperature of the particle is equal to the saturation steam temperature and evaporation takes place at the surface and inside the particle. The vapor is driven out by a pressure gradient to the surface. We assume that the air, initially present in the particle, is completely removed when the sphere is heated to the saturation temperature.

The system of equations developed by Whitaker is the following: ${ }^{[15-17]}$

- Mass conservation equation in the liquid phase:

$$
\frac{\partial\left\langle\varepsilon_{1} \rho_{1}\right\rangle}{\partial \mathrm{t}}+\nabla \cdot\left\langle\rho_{1}\right\rangle\left\langle\mathrm{U}_{1}\right\rangle=-\dot{\mathrm{m}}_{\mathrm{v}}
$$

- Mass conservation equation in the gas phase:

$$
\frac{\partial\left\langle\rho_{\mathrm{g}}\right\rangle}{\partial \mathrm{t}}+\nabla \cdot\left[\left\langle\rho_{\mathrm{g}}\right\rangle^{\mathrm{g}}\left\langle\mathrm{U}_{\mathrm{g}}\right\rangle\right]=\dot{\mathrm{m}}
$$

For an arbitrary $\alpha$-phase scalar or tensor variable $\Psi_{\alpha}$, we note:

- Superficial average:

$$
\left\langle\Psi_{\alpha}\right\rangle=\frac{1}{\mathrm{v}} \int_{\mathrm{v}_{\alpha}(\mathrm{t})} \Psi_{\alpha} \mathrm{dv}
$$

- Intrinsic average:

$$
\left\langle\Psi_{\alpha}\right\rangle^{\alpha}=\frac{1}{\mathrm{~V}_{\alpha}(\mathrm{t})} \int_{\mathrm{V}_{\alpha}(t)} \Psi_{\alpha} \mathrm{dv}
$$


The superficial and intrinsic averages are related by:

$$
\left\langle\Psi_{\alpha}\right\rangle=\varepsilon_{\alpha}\left\langle\Psi_{\alpha}\right\rangle^{\alpha}
$$

where $\varepsilon_{\alpha}=\mathrm{V}_{\alpha} / \mathrm{V}$ is the $\alpha$ phase volume fraction, or porosity.

- Darcy's law:

- The generalized Darcy's law is actually the only tool that can describe multi-phase stream in porous media. ${ }^{[20]}$ The average velocities of the liquid phase $\mathrm{U}_{1}$ and of the gas phase $\mathrm{U}_{\mathrm{g}}$ are obtained using relative permeability as following:

- Liquid phase:

$$
\left\langle\mathrm{U}_{1}\right\rangle=-\frac{\mathrm{KK}_{\mathrm{r} 1}}{\mu_{1}}\left(\nabla\left\langle\mathrm{P}_{1}\right\rangle^{1}-\rho_{\mathrm{l}} \mathrm{g}\right)
$$

with:

$$
\mathrm{P}_{\mathrm{c}}=\left\langle\mathrm{P}_{\mathrm{g}}\right\rangle^{\mathrm{g}}-\left\langle\mathrm{P}_{1}\right\rangle^{1}
$$

Gas phase:

$$
\left\langle\mathrm{U}_{\mathrm{g}}\right\rangle=-\frac{\mathrm{KK}_{\mathrm{rg}}}{\mu_{\mathrm{g}}}\left(\nabla\left\langle\mathrm{P}_{\mathrm{g}}\right\rangle^{\mathrm{g}}-\left\langle\rho_{\mathrm{g}}\right)^{\mathrm{g}} \mathrm{g}\right)
$$

where $\left\langle\mathrm{P}_{\mathrm{l}}\right\rangle^{1}$ and $\left\langle\mathrm{P}_{\mathrm{g}}\right\rangle^{\mathrm{g}}$ are the intrinsic average pressures of the liquid phase and the gas phase, respectively. $\mathrm{K}$ is the intrinsic permeability and $\mathrm{K}_{\mathrm{rg}}$ and $\mathrm{K}_{\mathrm{rl}}$ are the relative permeability of the gas phase and the liquid phase, respectively. Pressures in the liquid and gaseous phases are related to the capillary pressure $\mathrm{P}_{\mathrm{c}}$ considered as a characteristic property of the porous medium.

Assuming the specific heats are constant and using the mass conservation equation, the energy conservation equation is

$$
\begin{aligned}
& \left\langle\rho \mathrm{C}_{\mathrm{p}}\right\rangle \frac{\partial\langle\mathrm{T}\rangle}{\partial \mathrm{t}}+\left(\mathrm{C}_{\mathrm{pl}}\langle\rho\rangle_{1}^{1}\langle\mathrm{U}\rangle_{1}^{1}+\mathrm{C}_{\mathrm{pv}}\langle\rho\rangle_{\mathrm{v}}^{\mathrm{v}}\langle\mathrm{U}\rangle_{\mathrm{v}}^{\mathrm{v}}\right) \nabla \mathrm{T} \\
& =\nabla \cdot\left(\lambda_{\mathrm{eff}} \nabla\langle\mathrm{T}\rangle\right)-\Delta \mathrm{H}_{\mathrm{v}} \cdot \dot{\mathrm{m}}_{\mathrm{v}}
\end{aligned}
$$

where $\left\langle\rho \mathrm{C}_{\mathrm{p}}\right\rangle$ is the constant pressure heat capacity of the porous particle.

Mass conservation of water in the porous particle:

$$
\begin{aligned}
& \rho_{\mathrm{s}} \frac{\partial \mathrm{X}}{\partial \mathrm{t}}= \\
& \nabla \cdot\left\{\begin{array}{l}
\left(\left(\rho_{1} \frac{\mathrm{KK}_{\mathrm{rv}}}{\mu_{\mathrm{l}}}+\rho_{\mathrm{v}} \frac{\mathrm{KK}_{\mathrm{rv}}}{\mu_{\mathrm{v}}}\right) \frac{\partial \mathbf{P}_{\mathrm{g}}}{\partial \mathrm{X}}-\rho_{1} \frac{\mathrm{KK}_{\mathrm{rl}}}{\mu_{1}} \frac{\partial \mathbf{P}_{\mathrm{c}}}{\partial \mathrm{X}}\right) \nabla \mathrm{X} \\
+\left(\left(\rho_{\mathrm{v}} \frac{\mathrm{KK}_{\mathrm{rv}}}{\mu_{\mathrm{v}}}+\rho_{1} \frac{\mathrm{KK}_{\mathrm{rl}}}{\mu_{1}}\right) \frac{\partial \mathbf{P}_{\mathrm{g}}}{\partial \mathrm{T}}-\rho_{1} \frac{\mathrm{KK}_{\mathrm{rl}}}{\mu_{1}} \frac{\partial \mathbf{P}_{\mathrm{c}}}{\partial \mathrm{T}}\right) \nabla \mathrm{T}
\end{array}\right\}
\end{aligned}
$$

\section{Thermodynamic Relations}

- The partial pressure of vapor is equal to its equilibrium pressure:

$$
\mathrm{P}_{\mathrm{v}}=\mathrm{P}_{\mathrm{veq}}(\mathrm{T}, \mathrm{S})
$$

where $\mathrm{S}$ is the saturation.

- The gaseous phase is assumed to consist of ideal gas.

$$
\left\langle\mathrm{P}_{\mathrm{g}}\right\rangle=\frac{\left\langle\rho_{\mathrm{g}}\right\rangle \mathrm{R}\langle\mathrm{T}\rangle}{\mathbf{M}_{\mathrm{g}}}
$$

\section{Initial Conditions}

The initial heating of the material is not included in this model. It is assumed that the initial temperature and pressure of the material are uniform and equal to the saturation temperature and pressure of the surrounding gas. Hence, early condensation of steam was not considered in the simulation.

\section{Boundary Conditions}

The number of the possible exchange configurations and the lack of understanding of the transfer mechanism at the product surface and surrounding drying agent lead to major numerical difficulties. Particularly, the mass transfer at the interface becomes difficult to evaluate when the surface is covered by a liquid film. We assume that the transfer of humidity at the surface takes place only in the vapor phase.

At the particle surface, the continuity of energy and mass flux allows:

$$
\begin{gathered}
\left(\lambda_{\mathrm{eff}} \nabla\langle\mathrm{T}\rangle+\Delta \mathrm{H}_{\mathrm{v}}\left\langle\rho_{1}\right\rangle^{1}\left\langle\mathbf{U}_{1}\right\rangle^{1}\right) \cdot \mathbf{n}=\mathrm{h}\left(\langle\mathrm{T}\rangle-\mathrm{T}_{\infty}\right) \\
\left(\left\langle\rho_{1}\right\rangle^{1}\left\langle\mathrm{U}_{1}\right\rangle^{1}+\Delta \mathrm{H}_{\mathrm{v}}\left\langle\rho_{\mathrm{v}}\right\rangle^{\mathrm{v}}\left\langle\mathrm{U}_{\mathrm{v}}\right\rangle^{\mathrm{v}}\right) \cdot \mathbf{n}=\mathrm{F}_{\mathrm{m}}
\end{gathered}
$$

where $\mathrm{h}$ is the convective heat transfer coefficient, $\mathrm{F}_{\mathrm{m}}$ is the mass flux, and $\mathbf{n}$ is the unit normal vector.

During drying at high temperature with superheated steam and humid air, the radiative transfer is not negligible. To introduce the radiative flux, we use the classical linearization of the total flux exchanged according to the surface temperature. This yields a global combined exchange coefficient (convection and radiation).

For the mass conservation equation, the natural physical condition at the drying surface is given by the equality of the partial vapor pressure and the external pressure.

$$
\mathrm{P}_{\mathrm{vsurf}}=\mathrm{a}_{\mathrm{w}}(\mathrm{X}, \mathrm{T}) \mathrm{P}_{\mathrm{vsat}}(\mathrm{T})=\mathrm{P}_{\infty}
$$

where the saturation pressure of the vapor phase is given by: ${ }^{[21]}$

$$
\mathrm{P}_{\mathrm{vsat}}(\mathrm{T})=\exp \left(25.5058-\left(\frac{5204.9}{\mathrm{~T}}\right)\right)
$$


The natural boundary condition for mass transport is given by Eq. (15). This condition must be expressed in terms of mass flux (Eq. (14)). The system is then solved with an iterative method where the value of the mass flux should fulfill the boundary condition of the partial pressure of the vapor. It can be noticed that the vapor pressure at the product surface is a decreasing function of the evaporation mass flux. A full description of the iterative method is given in Couture. ${ }^{[22]}$

When superheated steam is the drying medium, mass transfer resistance at the interface is negligible, and the heat flux received by convection at the interface between the porous particle surface from the surrounding steam in the constant rate period is

$$
\mathrm{F}_{\max }=\frac{\mathrm{h}\left(\mathrm{T}_{\text {surf }}-\mathrm{T}_{\infty}\right)}{\Delta \mathrm{H}_{\mathrm{v}}}
$$

where $T_{\text {surf }}$ is the temperature at the particle surface, $T_{\infty}$ is the steam temperature, and $\mathrm{h}$ is the heat transfer coefficient given by:

$$
\mathrm{h}=\frac{\mathrm{Nu} \lambda_{\mathrm{g}}}{\mathrm{d}}
$$

For the convective heat transfer, we used the correlation proposed by Hager et al.:. ${ }^{[18]}$

$$
\mathrm{Nu}=2+0.61 \operatorname{Re}^{0.52} \operatorname{Pr}^{0.33}
$$

The constant rate period begins when the surface of the product reaches the saturation temperature. As long as the surface remains at the saturation temperature, the drying rate is constant and controlled by the heat transfer rate at the interface. When the surface temperature rises above the saturation temperature, the constant rate period is achieved.

The main difference between the formulation of superheated steam drying and humid air drying is the expression of mass flux. Indeed, Eq. (15) is not applicable for humid air drying because $\mathrm{P}_{\mathrm{vsurf}}$ is not equal to $\mathrm{P}_{\mathrm{v} \infty}$. So in this case, the boundary condition at the particle external surface is:

$$
F_{m}=\frac{h_{m} M}{R}\left(\frac{P_{\text {vsurf }}}{T_{\text {surf }}}-\frac{P_{v_{\infty}}}{T_{\infty}}\right)
$$

where $h_{m}$ is the mass transfer coefficient:

$$
\mathrm{h}_{\mathrm{m}}=\frac{\mathrm{D}_{\mathrm{m}}}{\mathrm{d}} \mathrm{Sh}
$$

$\mathrm{P}_{\mathrm{vsurf}}$ and $\mathrm{P}_{\mathrm{v} \infty}$ are the vapor pressure at the particle surface and the vapor pressure of the drying agent, respectively. The humid air is assumed to consist of ideal gas.

To calculate the mass transfer coefficient, we used the relation presented by Ranz: ${ }^{[23]}$

$$
\mathrm{Sh}=2+0.6 \mathrm{Re}^{1 / 2} \mathrm{Sc}^{1 / 3}
$$

where $\mathrm{D}_{\mathrm{m}}$ is the molecular diffusion coefficient of vapor in the gas and $\mu_{\mathrm{g}}$ and $\rho_{\mathrm{g}}$ are the dynamic viscosity and the density of the gas, respectively.

\section{Numerical Resolution}

The system of equations is solved by a finite volume method based on the notion of a control domain ${ }^{[24]}$ with a nonregular mesh. To insure the convergence, an upwind scheme is used to evaluate convection terms on faces of the control domain. The discretization of the conservation equations leads to a system of algebraic equations. This system is strongly coupled and is solved numerically by the iterative method of Gauss Seidel.

\section{RESULTS AND DISCUSSION}

\section{Comparison with Experimental Data}

The model is validated with experimental data reported in the literature. ${ }^{[18,19]}$ Hager et al. ${ }^{[18]}$ investigated experimentally the superheated steam drying of a single porous particle of ceramic. These spheres, with $10 \mathrm{~mm}$ of diameter, were exposed to superheated steam flowing through a chamber with an inner diameter of $16 \mathrm{~mm}$. The characteristics of the ceramic porous particle used in the simulation are listed in Table 1. The particle temperature and the evaporation rate were measured as a function of time. Figure 1 shows the comparison of the model results with the experimental data under different steam temperatures and gas mass fluxes. The experimental data of Looi et al. ${ }^{[19]}$ was also used for the validation of the model. In this report, Looi et al. ${ }^{[19]}$ have investigated the superheated steam drying of porous ceramic particles under pressure. The inside diameter and the length of the drying chamber were approximately 25 and $100 \mathrm{~mm}$, respectively. During each experiment, they inserted two porous particles $(\mathrm{d}=9.9 \mathrm{~mm})$ in the drying chamber, one of them to measure the center particle temperature with thermocouple, and the second particle was placed in a wire basket that was suspended to a force transducer. During drying, the particle mass was measured using an industrial load cell. The properties of the ceramic porous particle used in the simulation are shown in Table 1. Figure 2 illustrates the comparison of predicted and experimental data. We notice that in these figures, the predicted temperature rises faster than the experimental one. However, the time needed to reach the drying temperature is equal. The model predictions are clearly in good agreement with the experiments. The discrepancies between predicted and measured solid temperature can be attributed to the position of the thermocouples and errors in the measurement.

The model of Hager et al. ${ }^{[18]}$ is based on the thermodynamic approach improved by Moyne et al. ${ }^{[20]}$ They considered the effect of gravity and pressure gradient. The phenomenological coefficients intervening in equations 
TABLE 1

Properties of the porous ceramic and coal particles used in the simulation. These parameter are given in the literature $\mathrm{e}^{[10,17,18,21]}$

\begin{tabular}{lccc}
\hline Parameters & Ceramic spheres (Fig. 1) & Ceramic spheres (Fig. 2) & Coal (Figs. 10, 11, 12) \\
\hline $\mathrm{d}(\mathrm{mm})$ & 10 & 9.9 & 10 \\
$\rho_{\mathrm{s}}\left(\mathrm{kg} / \mathrm{m}^{3}\right)$ & 2000 & 1550 & 1100 \\
$\varepsilon$ & 0.4 & 0.41 & 0.4 \\
$\mathrm{C}_{\mathrm{ps}}(\mathrm{J} / \mathrm{kg} \mathrm{K})$ & 640 & 880 & 1000 \\
$\lambda_{\mathrm{s}}(\mathrm{W} / \mathrm{m} \mathrm{K})$ & 1.7 & 1.3 & 2.5 \\
$\mathrm{~K}\left(\mathrm{~m}^{2}\right)$ & $2 \times 10^{-15}$ & $2 \times 10^{-15}$ & $2 \times 10^{-15}$ \\
$\mathrm{~K}_{\mathrm{rl}}$ & $\mathrm{S}^{2}$ & $\mathrm{~S}^{2}$ & $\mathrm{~S}^{2}$ \\
$\mathrm{~K}_{\mathrm{rg}}$ & $(1-\mathrm{S})^{2}$ & $(1-\mathrm{S})^{2}$ & $(1-\mathrm{S})^{2}$ \\
$\mathrm{X}_{\mathrm{i}}(\mathrm{kg} / \mathrm{kg})$ & 0.184 & 0.223 & 1 \\
$\rho_{1}\left(\mathrm{~kg} / \mathrm{m}^{3}\right)$ & 4180 & 4180 & 4180 \\
\hline
\end{tabular}

were determined by experiment or theory. This is the disadvantage of this model because the determination of these coefficients is complicated. Looi et al. ${ }^{[19]}$ developed a model based on a simple receding core. The evaporation of moisture occurs only on a defined drying front. They assumed that mass transfer resistance and radiative heat transfer are negligible. Heat is transferred from the surrounding superheated steam to the particle surface by

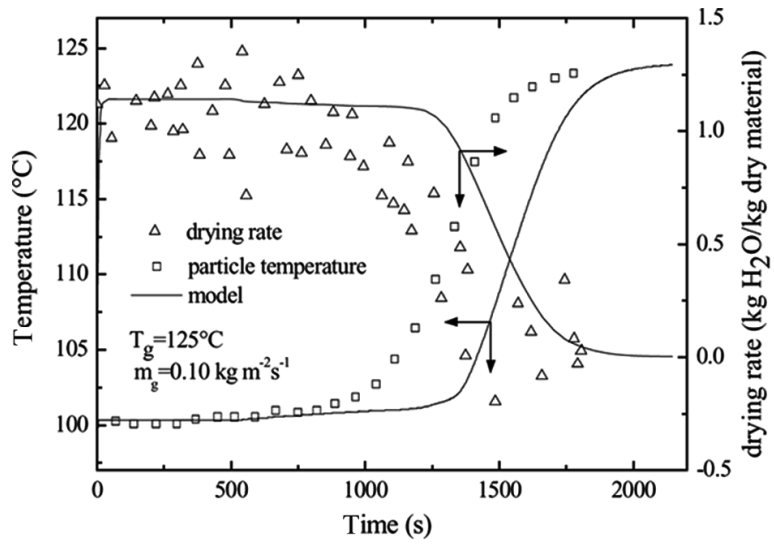

(a)

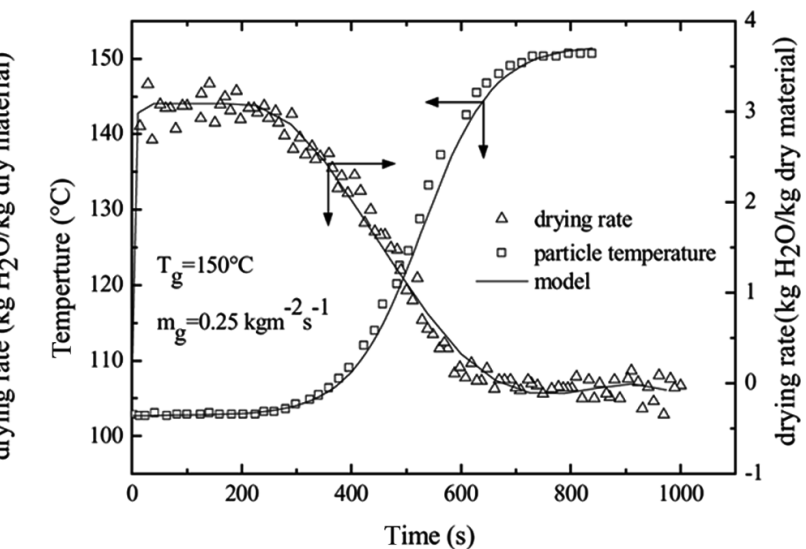

(b)

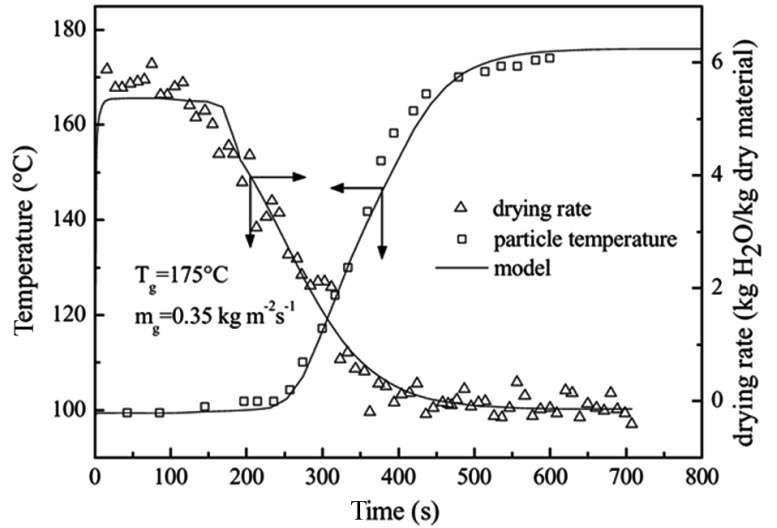

(c)

FIG. 1. Comparison of model with experimental data of spherical ceramic porous particle. ${ }^{[17]}$ 


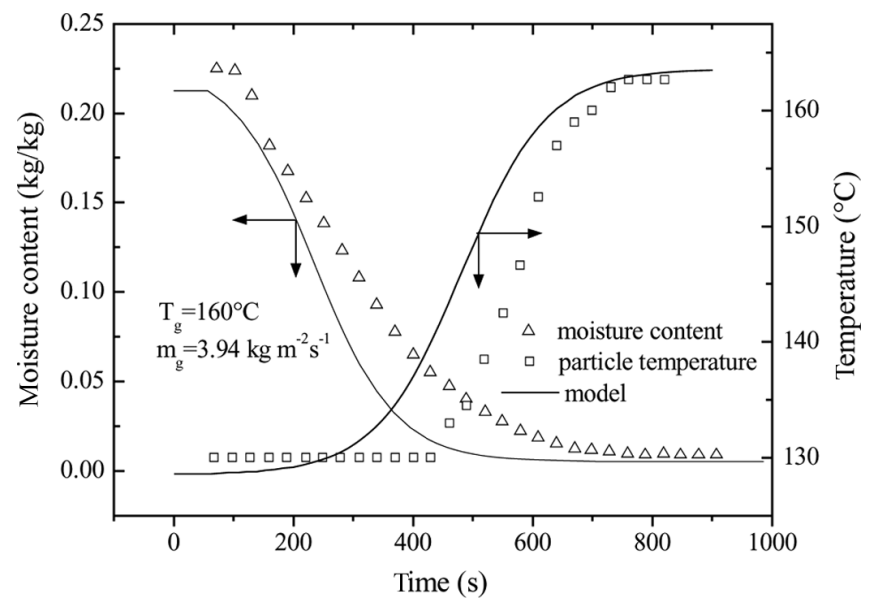

FIG. 2. Comparison of model with experimental data of spherical ceramic porous particle. ${ }^{[18]}$

convection. Our model is based on the volume averaging method and, as mentioned above, an iterative process is used for the calculation of the mass flux, verifying the boundary condition. Thus, only the knowledge of the physical properties of the porous medium are needed. The agreement found between experimental and predicted results indicates that using the volume averaging method to describe heat and mass transfer during the drying of a porous particle is successful.

\section{Sensitivity Analysis}

The sensitivity analysis indicated that variations in certain parameters have significant effects on the drying time. Knowledge of the parameters that have significant impact on drying behavior is useful in designing drying operations.

Figure 3 illustrates the effect of particle diameter on the drying process. We noted that particle diameter exerts a significant influence on the drying behavior. Larger particles require a much longer time to reach the gas temperature due to the fact that more energy is needed to heat them up. We also note that for the same drying temperatures and steam velocities, the predicted moisture content decreases much more rapidly when the diameter decreases. This is due to the fact that the Reynolds number and thus the heat transfer coefficient increases with smaller particles. Figure 4 illustrates how changes in the temperature of the drying medium affect the drying curve. At lower temperatures, the moisture content decreases more slowly. As the temperature increases, the medium reaches the drying temperature more quickly. This is because the higher temperature gradient in the medium resulted in a higher heat flux. ${ }^{[25]}$

The gas mass flux is related to the gas velocity via the gas density. As we would expect, when we increase the gas mass flux, for the same drying temperature, the Reynolds

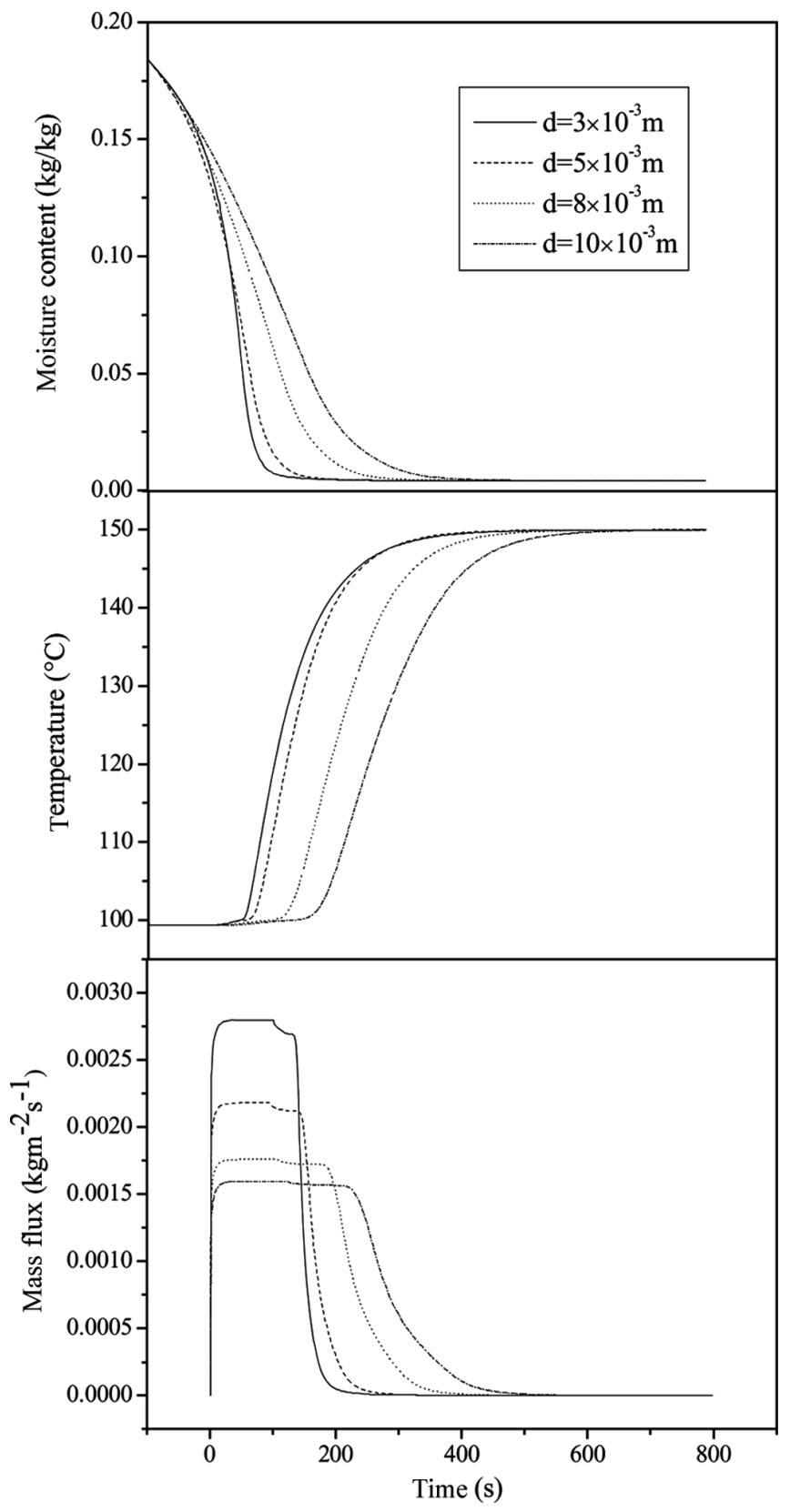

FIG. 3. Effect of particle diameter during superheated steam drying: $\mathrm{T}_{\mathrm{g}}=423 \mathrm{~K}$ and $\mathrm{m}_{\mathrm{g}}=0.78 \mathrm{kgm}^{-2} \mathrm{~s}^{-1}$.

number increases and consequently the convective heat transfer coefficient increases (Fig. 5) leading to a reduction of the drying time. Figure 6 shows the effect of permeability on the drying behavior of the single particle of ceramic, varying between $10^{-17}$ and $10^{-14} \mathrm{~m}^{2}$. It does not influence the value of the heat flux in the constant drying rate period but it affects the shape of falling rate phase. However, the time required to reach the equilibrium moisture content increases as the permeability decreases. 


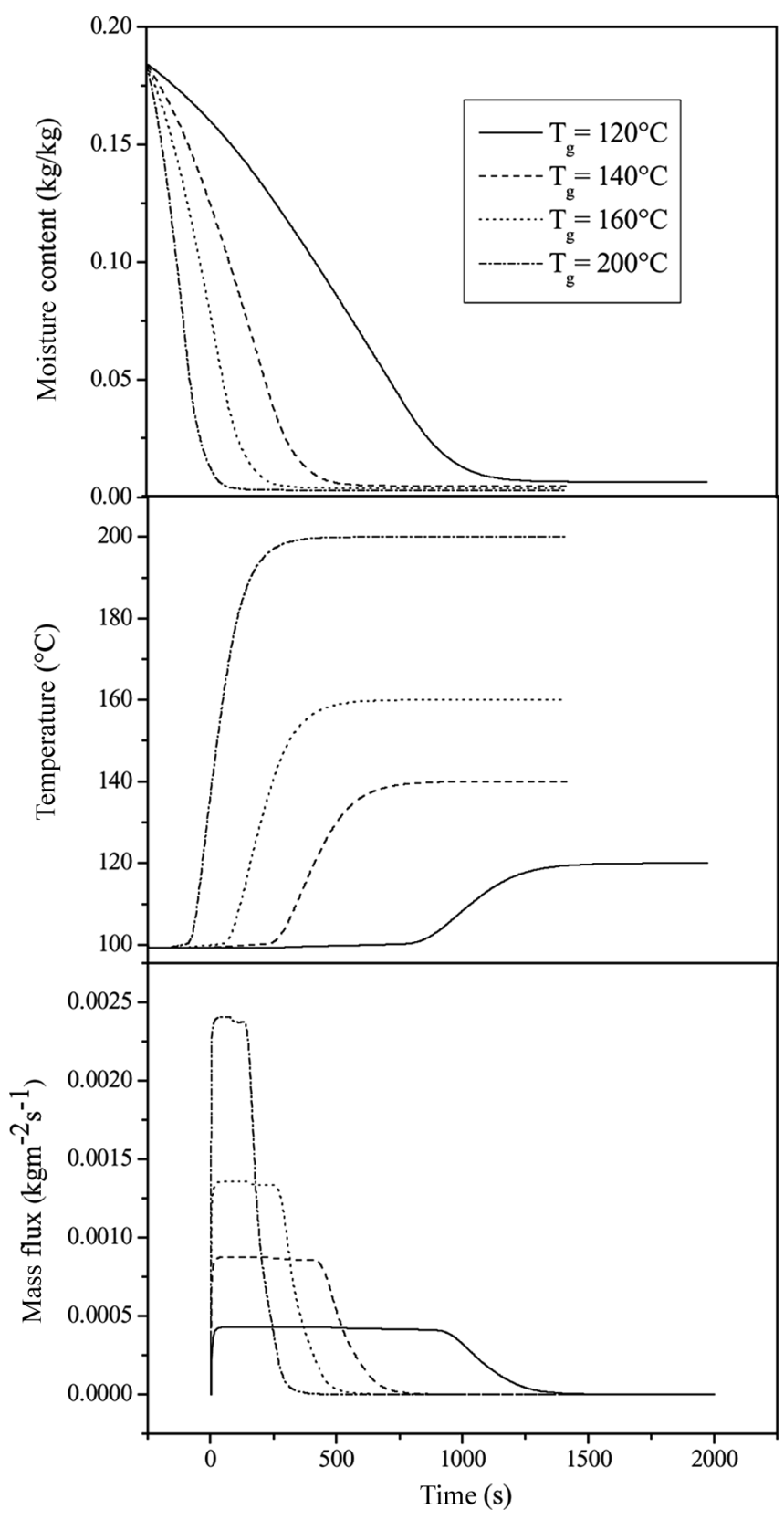

FIG. 4. Effect of gas temperature during superheated steam drying: $\mathrm{m}_{\mathrm{g}}=0.26 \mathrm{kgm}^{-2} \mathrm{~s}^{-1}$ and $\mathrm{d}=10 \mathrm{~mm}$.

In the range of variables considered, steam temperature exerts the most significant effect on the drying behavior.

The model can be used to determine the inversion temperature. Figure 7 shows that the mass fluxes during superheated steam drying and humid air drying intersect at a point, the so-called inversion temperature, at which the mass fluxes of the two drying processes are equal. The inversion temperature of porous ceramic particle is about $152^{\circ} \mathrm{C}$. This value is lower than the reported value

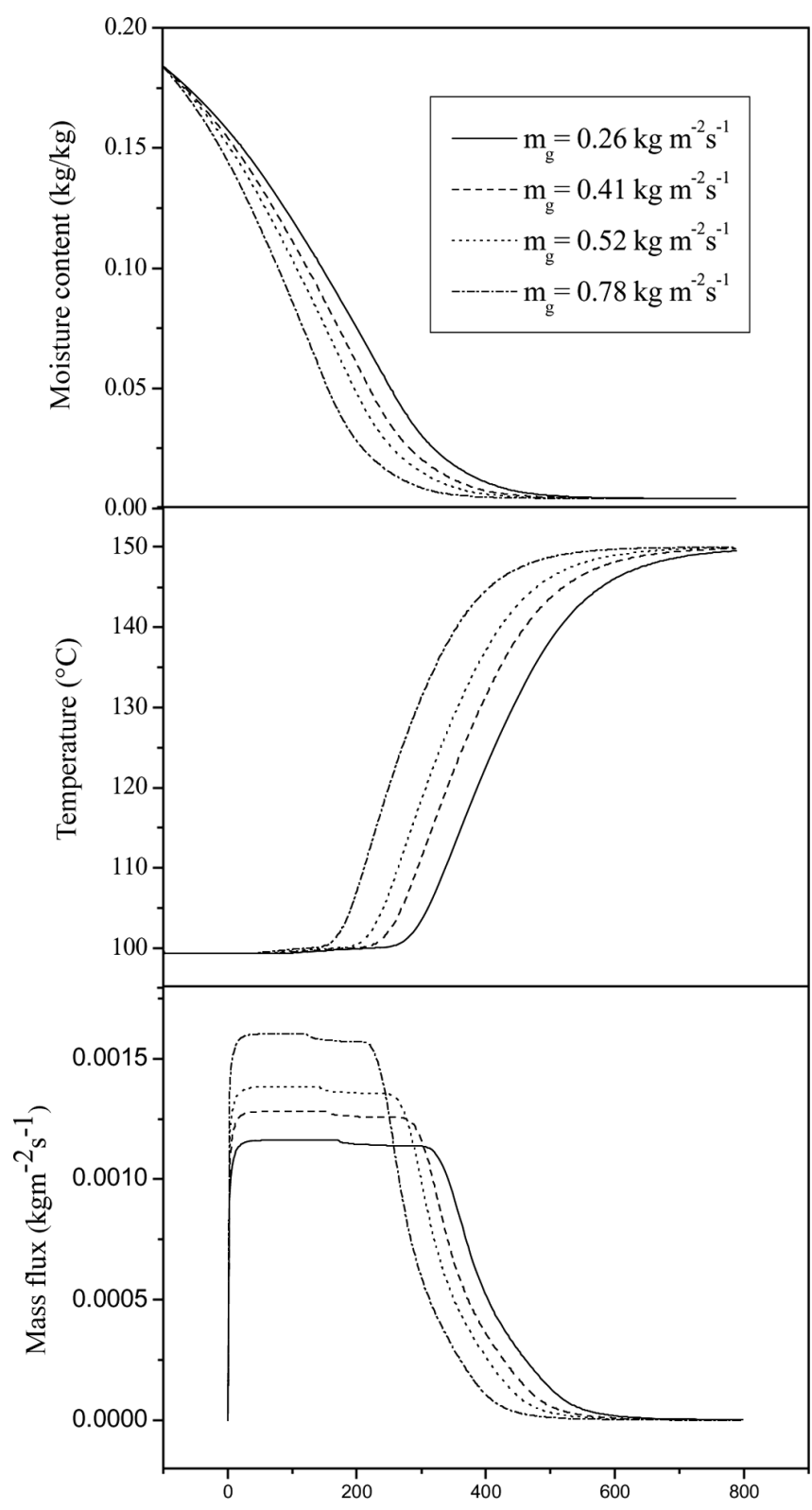

FIG. 5. Effect of gas mass flux during superheated steam drying: $\mathrm{T}_{\mathrm{g}}=423 \mathrm{~K}$ and $\mathrm{d}=10 \mathrm{~mm}$.

of Tatemoto et al. ${ }^{[14]}\left(160^{\circ} \mathrm{C}\right)$ for ceramic plate drying. They defined the inversion temperature as the temperature at which the drying times for the superheated steam and hot air drying processes are equal. The drying time was also defined as the time when the drying rate becomes zero. They assumed that convective gas transfer in the sample is considered by using Blake-Kozeny equation. ${ }^{[14]}$

The effects of various operating parameters on the inversion temperature for the drying of porous ceramic spheres were investigated using the mathematical model 


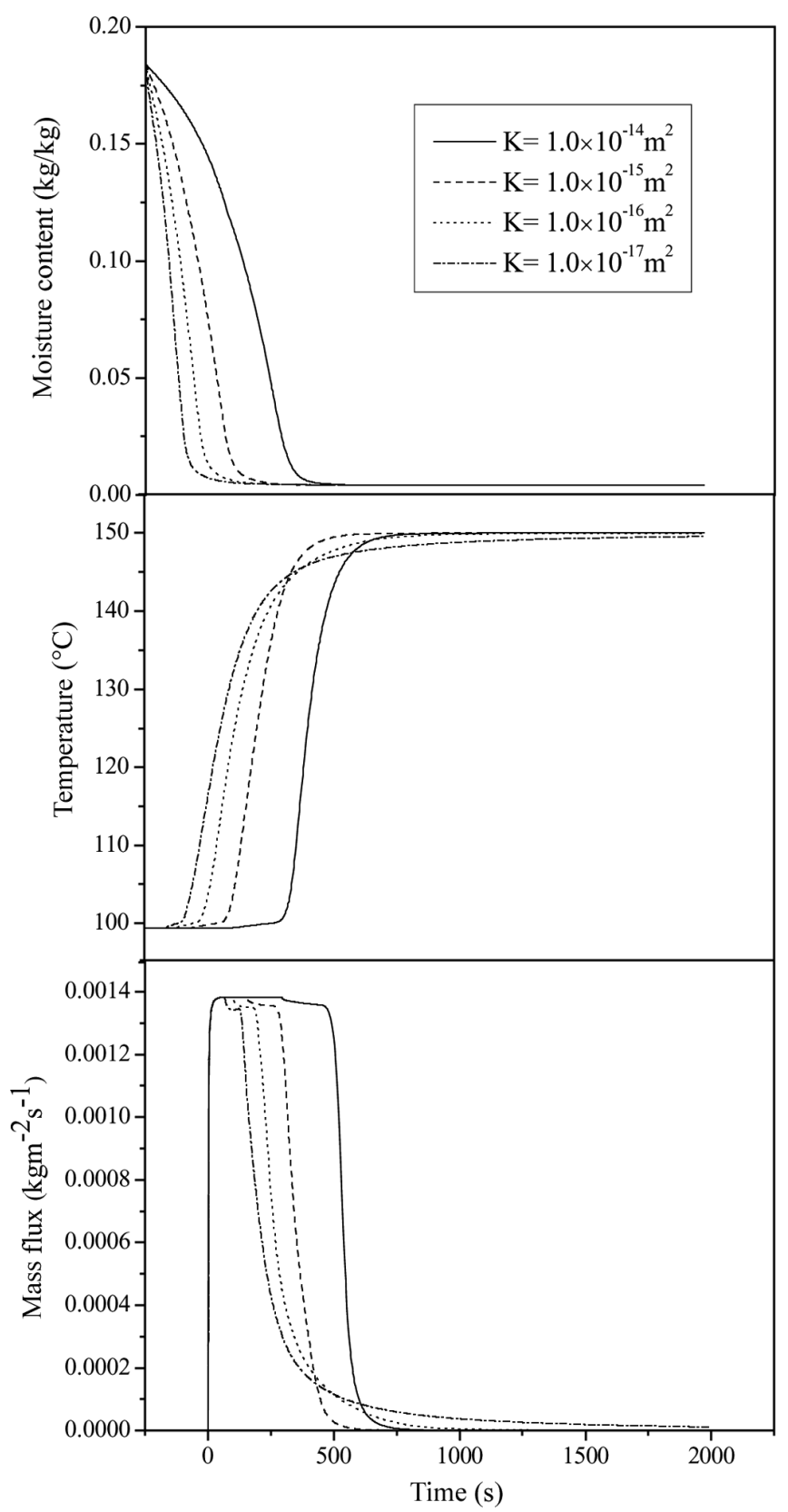

FIG. 6. Effect of permeability during superheated steam drying: $\mathrm{T}_{\mathrm{g}}=423 \mathrm{~K}, \mathrm{~m}_{\mathrm{g}}=0.1 \mathrm{kgm}^{-2} \mathrm{~s}^{-1}$, and $\mathrm{d}=2 \mathrm{~mm}$.

developed in the previous section. Figure 8 shows the influence of the gas mass flux and the particle diameter on the inversion temperature for the drying of a porous spherical ceramic particle. The inversion temperature is calculated during the constant rate period. We note the existence of a minimum as the gas mass flux and particle diameter increase. Chen et al. ${ }^{[1]}$ reported the same behavior during coal drying. As shown in Fig. 6, permeability does not affect the value of mass flux in the constant rate period.

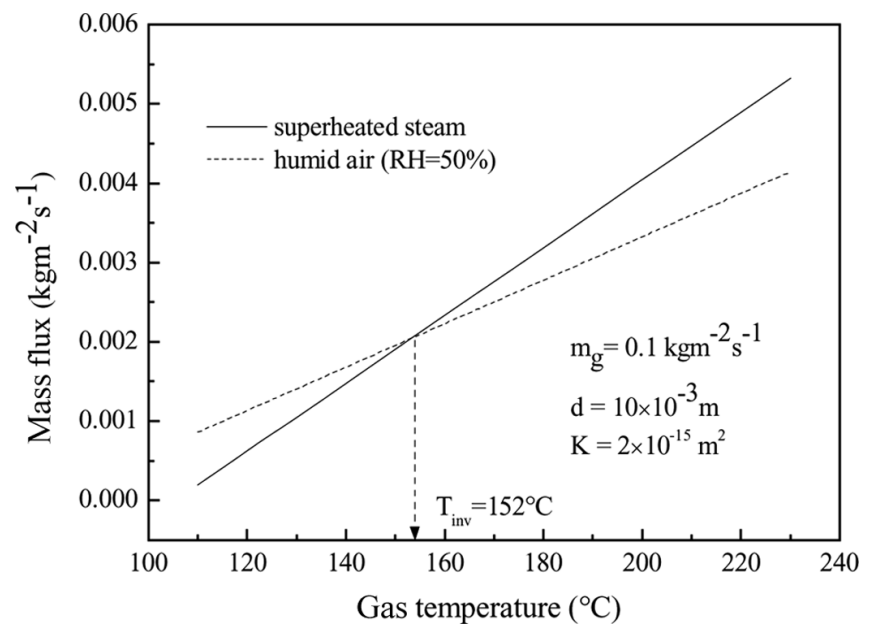

FIG. 7. Inversion temperature $\left(\mathrm{T}_{\mathrm{inv}}=425 \mathrm{~K}\right)$ : the intersection of the two curves.

Therefore, to investigate the effect of permeability, the inversion temperature is calculated only during the falling rate period. Figure 9 illustrates the effect of permeability on the inversion temperature. An increase in ceramic particle permeability leads to a decrease of the inversion temperature. In fact, an increase in permeability leads to a decrease in the drying time and an increase in the evaporation rate which leads to a decrease of the inversion temperature.

Next, we compare our model with that of Chen et al. ${ }^{[1]}$ for the coal inversion temperature. For this purpose, the same coal properties were used (Table 1). Figures 10, 11,

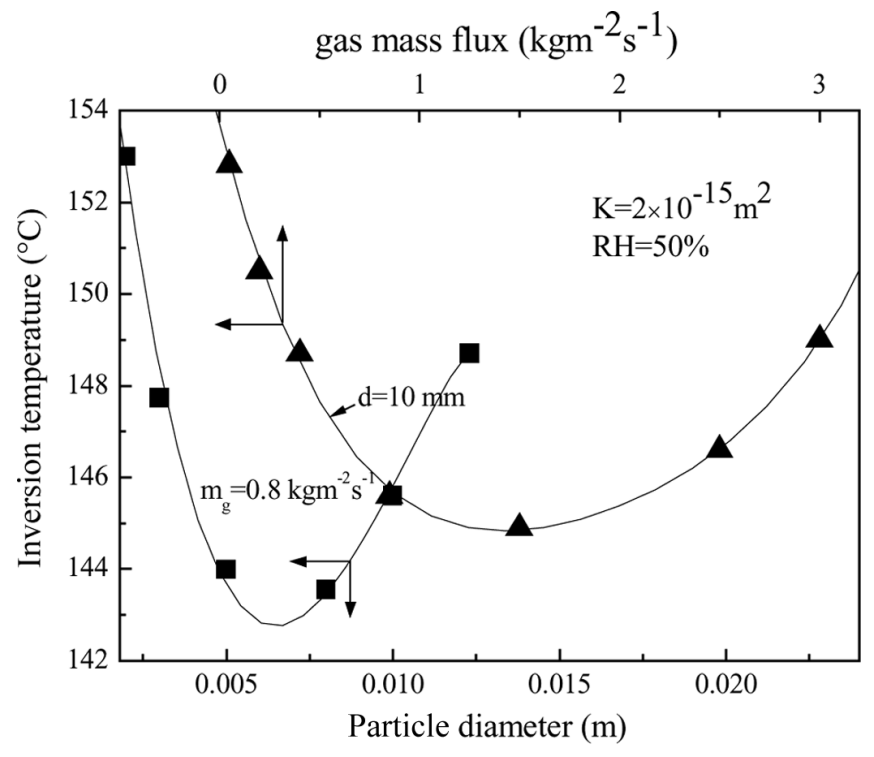

FIG. 8. Influence of gas mass flux and particle diameter on the inversion temperature. 


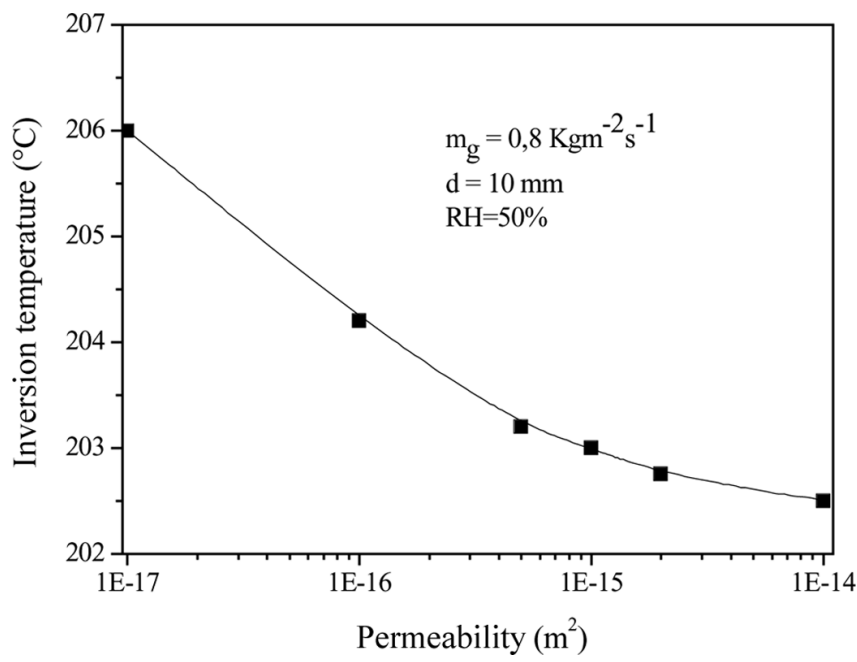

FIG. 9. Influence of the permeability on the inversion temperature.

and 12 show the results of the two models. The effect of the gas mass flux, particle radius, and permeability on the coal inversion temperature is illustrated. The same behavior between both models is observed; however, our results give slightly smaller values of the inversion temperature compared to Chen et al. The difference can be attributed to the fact that these authors used a front model, which assumes that the removal of water proceeds with the formation of a wet-dry interface and a receding front with time. Moreover, the condensation phase was considered since the initial temperature is smaller than the saturated temperature. Iyota et al. ${ }^{[26]}$ studied the effect of condensation during the initial stage of superheated steam drying. They demonstrated that this phenomenon influences the drying process and the quality of the dried solid.

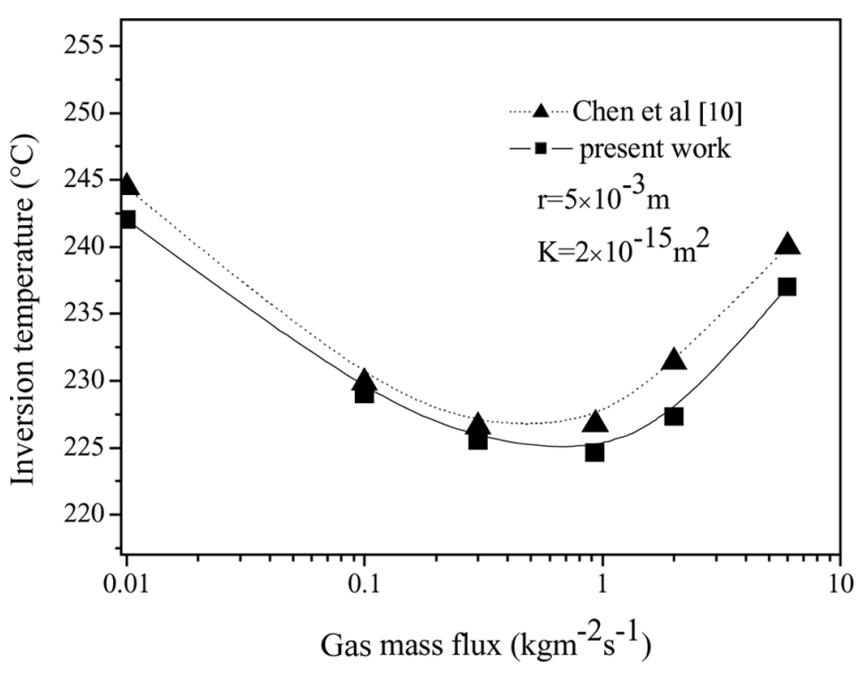

FIG. 10. Influence of gas mass flux on the coal inversion temperature.

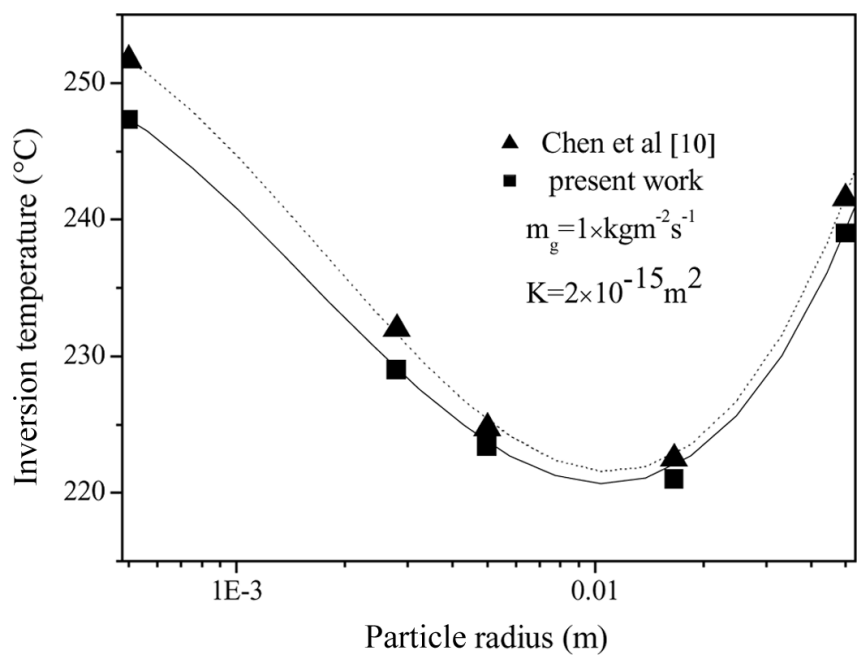

FIG. 11. Influence of particle radius on the coal inversion temperature.

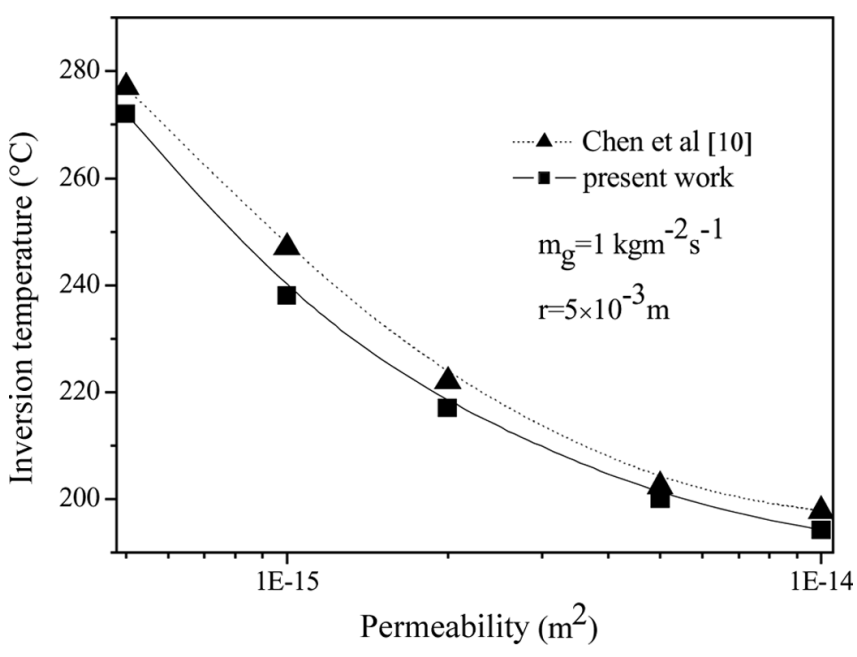

FIG. 12. Influence of permeability on the coal inversion temperature.

\section{CONCLUSION}

Single porous particle model describing heat and mass transfer has been developed to investigate the drying with superheated steam and humid air. The model is validated with literature experimental data of spherical porous ceramic particle. A good agreement is found between the simulation and experimental results. The extension of this model allows predicting an inversion temperature at which the evaporation rates into both superheated steam and humid air are equal. A value of about $152^{\circ} \mathrm{C}$ is obtained. The influence of external and internal parameters on the drying kinetics and on the inversion temperature is also investigated. As expected, the inversion temperature for the drying of porous particles depends on the physical 
properties of the porous medium like permeability and particle diameter and on the external operating parameters like the gas mass flux. Calculations illustrate that this temperature exhibits a minimum relative to the gas mass flux and particle diameter, and it decreases with the increase of the ceramic permeability. These results are in good agreement with similar calculations of the inversion temperature for coal drying.

\section{NOMENCLATURE}

$\mathrm{a}_{\mathrm{w}} \quad$ (Water activity)

$\mathrm{C}_{\mathrm{p}} \quad$ Constant pressure specific heat $\left(\mathrm{J} \mathrm{kg}^{-1} \mathrm{~K}^{-1}\right)$

$\mathrm{D}_{\mathrm{m}} \quad$ Molecular diffusion coefficient $\left(\mathrm{m}^{2} \mathrm{~s}^{-1}\right)$

d Particle diameter $(\mathrm{m})$

$\mathrm{F}_{\mathrm{m}} \quad$ Mass flux $\left(\mathrm{kg} \mathrm{m}^{-2} \mathrm{~s}^{-1}\right)$

$\mathrm{g} \quad$ Acceleration due to gravity $\left(\mathrm{m} \mathrm{s}^{-2}\right)$

$\Delta \mathrm{H} \quad$ Latent heat of vaporization $\left(\mathrm{J} \mathrm{kg}^{-1}\right)$

$\mathrm{h} \quad$ Heat transfer coefficient $\left(\mathrm{W} \mathrm{m}^{-2} \mathrm{~K}^{-1}\right)$

$\mathrm{h}_{\mathrm{m}} \quad$ Mass transfer coefficient $\left(\mathrm{m} \mathrm{s}^{-1}\right)$

$\mathrm{K} \quad$ Intrinsic permeability $\left(\mathrm{m}^{2}\right)$

$\mathrm{K}_{\mathrm{rg}} \quad$ Gas relative permeability

$\mathrm{K}_{\mathrm{rl}} \quad$ Liquid relative permeability

$\mathrm{K}_{\mathrm{rv}} \quad$ Vapor relative permeability

M Molecular weight $\left(\mathrm{kg} \mathrm{mol}^{-1}\right)$

$\dot{\mathrm{m}} \quad$ Evaporation rate $\left(\mathrm{kg} \mathrm{m}^{-3} \mathrm{~s}^{-1}\right)$

$\mathrm{m}_{\mathrm{g}} \quad$ Gas mass flux $\left(\mathrm{kg} \mathrm{m}^{-2} \mathrm{~s}^{-1}\right)$

n Unit normal vector

$\mathrm{P} \quad$ Pressure $(\mathrm{Pa})$

$\mathrm{P}_{\text {veq }} \quad$ Vapor equilibrium pressure $(\mathrm{Pa})$

$\mathrm{P}_{\text {vsat }} \quad$ Vapor saturation pressure $(\mathrm{Pa})$

$\mathrm{P}_{\mathrm{vsurf}} \quad$ Vapor pressure at particle surface $(\mathrm{Pa})$

$\mathrm{T}$ Temperature $\left({ }^{\circ} \mathrm{C}\right)$

t Time (s)

$\mathrm{R} \quad$ Universal gas constant $\left(\mathrm{Jmol}^{-1} \mathrm{~K}^{-1}\right)$

RH Relative humidity

$\mathrm{r} \quad$ Particle radius (m)

S Saturation

$\mathrm{U} \quad$ Velocity $\left(\mathrm{m} \mathrm{s}^{-1}\right)$

$\mathrm{V}_{\mathrm{g}} \quad$ Gas velocity $\left(\mathrm{m} \mathrm{s}^{-1}\right)$

X Moisture content (dry basis) $(\mathrm{kg} / \mathrm{kg})$

\section{Greek Symbols}

$\varepsilon \quad$ Porosity

$\varepsilon_{\mathrm{i}} \quad$ Volume fraction

$\lambda$ Thermal conductivity $\left(\mathrm{W} \mathrm{m}{ }^{-1} \mathrm{~K}^{-1}\right)$

$\mu \quad$ Dynamic viscosity ( $\mathrm{Pa} \mathrm{s}$ )

$\rho \quad$ Density $\left(\mathrm{kg} \mathrm{m}^{-3}\right)$

\section{Superscripts}

g Gas phase

$1 \quad$ Liquid phase

v Vapor

\section{Subscripts}

eq Equilibrium g Gas phase

I Initial surf

1 Liquid phase

m Mass

max Maximum

s Solid phase

sat Saturation

surf Surface

v Vapor

$\mathrm{v} \quad$ Vaporization

veq Vapor at equilibrium

$\infty \quad$ Gas agent

\section{Dimensionless Groups}

$\mathrm{Nu} \quad$ Nusselt number

Pr Prandtl number, $\operatorname{Pr}=\mu_{\mathrm{g}} \mathrm{C}_{\mathrm{pg}} / \lambda_{\mathrm{g}}$

$\mathrm{Re} \quad$ Reynolds number, $\mathrm{Re}=\rho_{\mathrm{g}} \mathrm{U}_{\mathrm{g}} \mathrm{d} / \mu_{\mathrm{g}}$

Sc $\quad$ Schmidt number, $\mathrm{Sc}=\mu_{\mathrm{g}} / \mathrm{D}_{\mathrm{m}} \rho_{\mathrm{g}}$

Sh Sherwood number

\section{REFERENCES}

1. Kumar, P.; Mujumdar, A.S. Superheated steam drying: A bibliography. Drying Technology 1990, 8 (1), 195-205.

2. Pronyk, C.; Cenkowski, S. Superheated steam drying technology. Paper number RRV03-0014, an ASAE Meeting Presentation, 2003.

3. Devahastin, S.; Suvarnakuta, P.; Soponronnarit, S.; Mujumdar, A.S A Comparative study of low-pressure superheated steam and vacuum drying of a heat-sensitive material. Drying Technology 2004, 22 (8), 1845-1867.

4. Iyota, H.; Nishimura, N.; Onuma, T.; Nomura, T.; 2001a, Drying of sliced raw potatoes in superheated steam and hot air. Drying Technology 2001, 19, 1411-1424.

5. Wenzel, L.; White, R.R. Drying granular solids in superheated steam. Industrial and Engineering Chemistry 1951, 43, 1829-1851.

6. Chu, J.C.; Lane, A.M.; Conklin, D. Evaporation of liquids into their superheated vapour. Industrial and Engineering Chemistry 1953, 45, 1586-1591.

7. Yoshida, T.; Hyodo, T. Evaporation of water in air humid air, and superheated steam. Industrial and Engineering Chemistry Process Design and Development 1970, 9, 207-214.

8. Trommelen, A.M.; Crosby, E.J. Evaporation and drying of drops in superheated vapours. AIChE Journal 1970, 857-867.

9. Schwartze, J.P.; Bröcker, S. A theoretical explanation of the inversion temperature. Chemical Engineering Journal 2002, 86, 61-67.

10. Costa, V.A.F.; Neto da Silva, F. On the rate of evaporation of water into a stream of dry air, humidified air and superheated steam, and the inversion temperature. International Journal of Heat and Mass Transfer 2003, 46, 3717-3726.

11. Chen, Z.; Wu, W.; Agarwal, P.K. steam drying of coal. Part 1. Modeling the behavior of a single particle. Fuel 2000, 79, 961-973.

12. Suvarnakuta, P.; Devahastin, S.; Soponronnarit, S.; Mujumdar, A.S. Drying kinetics and inversion temperature in a low-pressure superheated steam-drying system. Industrial and Engineering Chemistry Research 2005, 44, 1934-1941.

13. Shibata, H. Drying Rate curves of porous solids in steam and in air under low-pressure conditions. Drying Technology 2006, 24, 37-43.

14. Tatemoto, Y.; Bando, Y.; Oyama, K.; Yasuda, K.; Nakamura, M.; Sugimura, Y.; Shibata, M. Effects of operational conditions on drying characteristics in closed superheated steam drying. Drying Technology 2001, 19 (7), 1287-1303. 
15. Whitaker, S. Heat and mass transfer in granular porous media. Advances in Drying 1980, 1, 23-61.

16. Whitaker, S. Moisture Transport mechanisms during the drying of granular porous media. Proceeding of the Fourth International Drying Symposium 1984, 1, 31-42.

17. Whitaker, S. Improved constraints for the principle of local thermal equilibrium. Industrial and Engineering Chemistry Research 1991, 30, 983-997.

18. Hager, J.; Hermansson, M.; Wimmerstedt, R. Modelling steam drying of a single porous ceramic sphere: experiment and simulations. Chemical Engineering Science 1997, 52 (8), 1253-1264.

19. Looi, A.Y.; Golonka, K.; Rhodes, M. Drying kinetics of single porous particles in superheated steam under pressure. Chemical Engineering Journal 2002, 87, 329-338.

20. Moyne, C.; Stemmelen, D.; Degiovanni, A. Asymmetric drying of porous materials at high temperature: theoretical analysis and experiments. International Chemical Engineering 1990, 30, 654-670.
21. Nadeau, J.P.; Puiggali, J.R. Séchage: Des Processus Physiques Aux Procédés Industriels; Lavoisier Tec et Doc: Paris, 1995.

22. Couture, F. Modélisation fine d'un problème de séchage, développement d'outils adaptés. Phd Thesis, Université de Bordeaux I, 1995.

23. Ranz, W.E. Friction and transfer coefficients for single particles and packed beds. Chemical Engineering Progress 1952, 48 (5), 247-253.

24. Patankar, S.V. Numerical Heat Transfer and Fluid Flow; Hemisphere: Washington, DC, 1980

25. Elustondo, D.M.; Mujumdar, A.S.; Urbicain, M.J. Optimum operating conditions in drying foodstuffs with superheated steam. Drying Technology 2002, 20 (2), 381-402.

26. Iyota, H.; Nishimura, N.; Yoshida, M.; Nomura, T. Simulation of superheated steam drying considering initial steam condensation, Drying Technology 2001, 19, 1425-1440. 